\title{
P04-2-68 Poster session
}

\section{Simulated clinical study in pharmacodynamic evaluation of new drug}

\author{
Liu Jianxun, Ren Jianxun, Ren Junguo, Lin Chengren, Li Lei \\ Xiyuan hospital of China Academy of Chinese Medical Sciences, China
}

Background: The aim of drug discovery is to find the safe, stable, effective and quality compounds and Chinese Herbal Medicinal Ingredients. Pharmacodynamic study is one of the most important projects. Whether it is chemosynthetic new drug or active ingredient extracted from plants and organisms, the effect of drug is evaluated by different animal experiments and pharmacological parameters in pharmacodynamic study. However, the focus deviation from effects and mechanisms of drug due to difference in anatomic structure and physiological characteristics between animal and human in pharmacological study will induce to significant reduction of chance in new drug development from preclinical to clinical study.

Method: We propose idea and method of simulated clinical study in pharmacodynamics study of new drug. It recommend to establish animal model which is similar to the pathophysiological processes of different diseases by choosing appropriate experimental animals similar to physiological characteristics, drug metabolism and pathological status of human being. The method that observing dynamic changes of function in animals in a shorter time and acquiring a valuable data by noninvasive or minimally invasive way need to be similar to clinical research. The aim of method can eliminate interruption that is impossible or difficult to reduce in general study and provide more valuable bioinformation. Result: Pharmacological study of synthetic drug (MG53), biological drug (PC-SOD) and chinese materia medica preparation had been completed in simulated clinical models of mini-swine, dog and rat with myocardial ischemia and atherosclerosis. Results demonstrated that these models had significant changes of electrocardiogram, heart function, myocardial ischemia and cardiac enzymes which were relation to pathological processes of coronary heart disease in clinical. MG53, PC-SOD and chinese materia medica preparation could interrupt pathophysiological processes of myocardial ischemia by different ways.

Conclusion: By preclinical study of the different drugs, we can conclude that method of simulated clinical study can facilitate further development of new therapeutic agents. 